\title{
Models for Substructural Arithmetics
}

\author{
Greg Restall* \\ Philosophy Department \\ The University of Melbourne \\ restall@unimelb.edu.au \\ Received by David Sweeney \\ Published September 22, 2010
}

http://www.philosophy.unimelb.edu.au/ajl/2010

(C) 2010 Greg Restall

\begin{abstract}
This paper explores models for arithmetic in substructural logics. In the existing literature on substructural arithmetic, frame semantics for substructural logics are absent. We will start to fill in the picture in this paper by examining frame semantics for the substructural logics $\mathrm{C}$ (linear logic plus distribution), $\mathrm{R}$ (relevant logic) and CK (C plus weakening). The eventual goal is to find negation complete models for arithmetic in $\mathrm{R}$.
\end{abstract}

This paper is dedicated to my friend and mentor Professor Robert K. Meyer, who taught me the joys of Relevant Arithmetic and so much more.

\section{SUBSTRUCTURAL ARITHMETICS}

Consider the Peano axioms for arithmetic.

$$
\begin{aligned}
\text { Identity } & 0=0 \\
& \forall x \forall y(x=y \rightarrow y=x) \\
& \forall x \forall y \forall z(y=z \rightarrow(x=y \rightarrow x=z)) \\
\text { Successor } & \forall x \forall y\left(x^{\prime}=y^{\prime} \rightarrow x=y\right) \\
& \forall x \forall y\left(x=y \rightarrow x^{\prime}=y^{\prime}\right) \\
& \forall x\left(0 \neq x^{\prime}\right) \\
\text { Addition } & \forall x(x+0=x) \\
& \forall x \forall y\left(x+y^{\prime}=(x+y)^{\prime}\right) \\
\text { Multiplication } & \forall x(x 0=0) \\
& \forall x \forall y\left(x y^{\prime}=x y+x\right) \\
\text { Induction } & A(0), \forall x\left(A(x) \rightarrow A\left(x^{\prime}\right)\right) \vdash \forall x A(x)
\end{aligned}
$$

${ }^{*}$ Research supported through Australian Research Council Grant DPO556827. Thanks to Miscellanea Logica (http://www.miscellanea-logica.info/) for permission to republish this article. It first appeared in Miscellanea Logica volume VII I, edited by MARTA BílKová. 
Under the standard interpretation of the language (in which quantifiers range over natural numbers) these axioms ring true. In fact, they ring true independently of the theory of the conditional ' $\rightarrow$ ' is read. If it is a merely material conditional we have traditional Peano arithmetic. If it is an intuitionistic conditional, we have intuitionistic Peano arithmetic. If it is the conditional of the relevant logic $\mathrm{R}$, then we have relevant arithmetic, studied by Meyer [5, 6, 7]. If it is the conditional of a contraction free logic, then we have contraction free arithmetics [12, I3]. Each of these different arithmetical theories have their virtues. For any predicate logic X possessing the vocabulary $\forall, \rightarrow, \sim$, we will call the theory given by adding these axioms and rules to those of $\mathrm{X}$, " $\mathrm{X}^{\sharp}$."

Gödel has shown us that $\mathrm{K}^{\sharp}$ is not complete (where $\mathrm{K}$ is classical predicate logic). As a result, neither is $\mathrm{X}^{\sharp}$ for any logic $\mathrm{X}$ weaker than $\mathrm{K}$. One way to complete $\mathrm{K}^{\sharp}$ is to add the infinitary $\omega$ rule.

$$
A(0), A\left(0^{\prime}\right), A\left(0^{\prime \prime}\right), \ldots \vdash \forall x A(x)
$$

We will call the arithmetic given by replacing the induction rule from $X^{\sharp}$ by the $\omega$ rule, " $\mathrm{X}^{\sharp \sharp}$. 妌 $\mathrm{K}^{\sharp \sharp}$ is complete. Here is why: One can prove by induction on the complexity of $A$ that $A$ is provable or $\sim A$ is provable. The crucial steps are the base case-that $s=t$ is provable iff the terms $s$ and $t$ denote the same number, and $s \neq t$ is provable iff the terms denote a different number. The cases for conjunction, disjunction and negation are trivial. The $\omega$ rule means that we can show that if $A(n)$ is provable for every $n$, then $\forall x A$ is provable. And similarly, if $A(n)$ is not provable for every $n$, then $\sim A(n)$ is provable for some $n$, and hence $\sim \forall x A$ is probable. Hence we have completeness for all formulae.

So, if you think that $\mathrm{K}$ is a good account of the logic of quantifiers and connectives, then you have reason to believe that $\mathrm{K}^{\sharp \sharp}$ is true arithmetic. It is a complete theory, giving you a definite answer for every arithmetic question.

The same cannot be said for $\mathrm{X}^{\sharp \sharp}$ for other logics $\mathrm{X}$. The most you can show is that (given that $\mathrm{X}$ satisfies a few conditions $\left.{ }^{2}\right) \mathrm{X}^{\sharp \sharp}$ is extensionally complete. That is, if $A$ is a formula in the extensional (implication free) vocabulary, either $A$ or $\sim A$ is provable. The proof is the same induction as before 3

Now, if you endorse a logic other than $\mathrm{K}$-say $\mathrm{X}$-then you must answer this question: If $\mathrm{X}$ is the preferred logic, is $\mathrm{X}^{\sharp \sharp}$ a good theory of arithmetic? Take the case of $R^{\sharp}$. In this theory there is no proof of $0=2 \rightarrow 0=1,4$ and

\footnotetext{
${ }^{\mathrm{I}}$ It is simple to show that given the $\omega$ rule the induction rule follows.

${ }^{2}$ In particular, that $\mathrm{X}$ includes distributive lattice logic and a negation satisfying the de Morgan laws.

${ }^{3}$ This proof obtains even in the absence of the law of the excluded middle.

4There is no way to reason from $0=2$ to $0=1$ using arithmetic operations alone. Reasoning classically, you can import the theorem $0 \neq 2$ to deduce $0=2 \wedge 0 \neq 2$ from $0=2$ alone, and from that contradiction you can deduce $0=1$. But in a logic respecting relevance, this reasoning breaks down.
} 
no proof of $\sim(0=2 \rightarrow 0=1)$ either:5 However, it is a theorem of $R^{\sharp \sharp}$ that $(0=2 \rightarrow 0=1) \vee \sim(0=2 \rightarrow 0=1)$. One could rightly ask which disjunct is the case. In relevant arithmetic, is it true that if $0=2$ then $0=1$ ? $R^{\sharp \sharp}$ gives you no guidance on the issue.

One way to proceed is to attempt to extend arithmetic further. We ought try to find theories extending $\mathrm{X}^{\sharp \sharp}$, which "fill in the blanks" in one way or other. One simple way to do this is to move to $\mathrm{K}^{\sharp}$, but this is giving up the game of formalising a truly substructural arithmetic. To be sure, we can complete $\mathrm{X}^{\sharp \sharp}$ by adding theses such as $0=2 \rightarrow 0=1,0=3 \rightarrow 0=2$ and so on, but to do so seems to erase all of the distinctions we wish to draw. If the conditional is to indicate some kind of relevance, then it is surely more appropriate for our theory to say that $0=2 \rightarrow 0=1$ is false. We ought have $\sim(0=2 \rightarrow 0=1)$ as a claim in our arithmetic theory.

One way to construct such a complete and genuinely substructural arithmetic involves the use of metavaluations. Meyer and I have shown that the theory $\mathrm{E}^{\sharp \sharp}$ can be extended to a natural complete theory, which we call $\mathrm{TE}^{\sharp \sharp}$, for "true E arithmetic" [IO]. This construction uses the technique of metavaluations to define the Truths of TE ${ }^{\sharp \sharp}$ as follows:

- $\mathrm{s}=\mathrm{t}$ is True iff $\mathrm{s}=\mathrm{t}$.

- $A \wedge B$ is True iff both $A$ and B are True.

- $A \vee B$ is True iff either $A$ or B is True.

- $\sim A$ is True iff $A$ is not True.

- $A \rightarrow B$ is True iff $A \rightarrow B \in E^{\sharp \sharp}$ and furthermore, if $A$ is True, so is $B$.

The set of Truths so defined is a theory extending TE\# ${ }^{\sharp}$. It is genuinely substructural, since it contains theses such as $\sim(0=2 \rightarrow 0=1)$, due to the defining clause for negations. This technique produces a complete arithmetic, but it has one major shortcoming. It works for the logic $\mathrm{E}$ of entailment, but not for the logic $R$ of relevant implication. In $R, \sim A$ is equivalent to $A \rightarrow f$ for a particular proposition $f$. This means that we cannot both give negation the extensional clause above ( $\sim A$ is true iff $A$ is not) while at the same time giving implication the intensional clause $(A \rightarrow B$ is when not only if $A$ is true so is $B$, but when also $A \rightarrow B$ is in the theory $X^{\sharp \sharp)}$. So, if we wish to complete $R^{\sharp \sharp}$ or theories like it, we will need to use a different technique.

In this paper I will present a different technique for constructing models for substructural arithmetics. This technique will help us shed new light on the issue of the truths of substructural arithmetics, and it be able to provide a number of complete extensions of $R^{\sharp \sharp}$. It seems, however, that these complete extensions of $\mathrm{R}^{\sharp \sharp}$ are not completely satisfactory (we will be able to construct

${ }^{5}$ If there were, then $\mathrm{K}^{\sharp \sharp}$ would be inconsistent, as $\mathrm{R}^{\sharp \sharp}$ is a subtheory of $\mathrm{K}^{\sharp \sharp}$, and we can prove $0=2 \rightarrow 0=1$ in $K^{\sharp \sharp}$. 
a consistent, complete arithmetic theory in which $\sim(0=2 \rightarrow 0=3)$, and another in which $\sim(0=3 \rightarrow 0=2)$. However, the techniques discussed in this paper does not allow us to satisfy both of them in the one model. So, I will end this paper with suggestions for future work.

\section{FRAME SEMANTICS}

The extant literature about models for substructural arithmetic has focussed on the use of algebraic models for modelling arithmetics [9, [II. The idea is simple. We take a domain of objects, and a domain of truth values. The arithmetic function symbols are interpreted by functions on the domain of objects, and the predicate of identity is interpreted by a function mapping pairs of objects in the domain to truth values in the propositional structures. The richer structure of the algebraic models of the non-classical logics choice enables us to model arithmetics with unusual properties.

The approach of this paper is different. We will not use algebraic models of our logics. We will not use three valued matrices or ever more complex algebras in order to prove interesting results about arithmetics. Instead, we will use two different kinds of frame semantics for substructural logics [ [I5].

Frame semantics for logics like $\mathrm{R}$ are rather simple ${ }^{6}$ A reduced frame for a substructural logic is a quintuple $\mathfrak{F}=\langle P, g, R, \sqsubseteq, *\rangle$ satisfying the following conditions.

- $\mathrm{P}$ is a nonempty set of points, including a distinguished point $\mathrm{g}$.

- $\mathrm{R}$ is a ternary relation on $\mathrm{P}$.

- $\sqsubseteq$ is a partial order on $P$.

- $*$ is a one place operator on $\mathrm{P}$.

- $\mathrm{R}$ satisfies the tonicity requirements: If Rabc $\mathrm{a}^{\prime} \sqsubseteq \mathrm{a}, \mathrm{b}^{\prime} \sqsubseteq \mathrm{b}$ and $\mathrm{c} \sqsubseteq \mathrm{c}^{\prime}$ then $\mathrm{Ra}^{\prime} \mathrm{b}^{\prime} \mathrm{c}^{\prime}$.

- $g$ is an identity point of R: Rgab if and only if $a \sqsubseteq b$.

- $*$ is order inverting: If $\mathrm{a} \sqsubseteq \mathrm{b}$ then $\mathrm{b}^{*} \sqsubseteq \mathrm{a}^{*}$.

The propositional connectives are modelled on a frame by way of an evaluation $\Vdash$ relating points and propositions satisfying the following constraints:

- If $\mathrm{a} \Vdash A$ and $\mathrm{a} \sqsubseteq \mathrm{b}$ then $\mathrm{b} \Vdash A$ too.

- $a \Vdash A \wedge B$ if and only if $a \Vdash A$ and $a \Vdash B$.

${ }^{6}$ This frame semantics for $\mathrm{R}$ and its neighbours was introduced by Routley and Meyer [I6] I7] in the 1970s. For a general discussion closest to the presentation here, see An Introduction to Substructural Logics, Chapter II II5. 
- $a \Vdash A \vee B$ if and only if $a \Vdash A$ or $a \Vdash B$.

- $a \Vdash A \rightarrow B$ if and only if for each $b, c$ where Rabc, if $b \Vdash A$ then $c \Vdash B$.

- $a \Vdash \sim A$ if and only if $a^{*} \Downarrow A$.

Entailment on frames is truth preservation at all points. That is, $A \vdash_{\mathfrak{F}} B$ if and only if for each $a \in \mathfrak{F}$, if $a \Vdash A$ then $a \Vdash B$. The identity point $g$ is a witness for these entailments since we have the following semantic entailment result.

$$
A \vdash_{\mathfrak{F}} B \text { if and only if } g \Vdash A \rightarrow B \text { in } \mathfrak{F} \text {. }
$$

The logic of these frames is quite weak. To extend the logic to model more familiar substructural logics, conditions are imposed on $\mathrm{R}, *$ and $\sqsubseteq$. Here are some conditions and their corresponding entailments:

$$
\begin{array}{cc}
\mathrm{R}(\mathrm{ab}) \mathrm{cd} \Rightarrow \operatorname{Ra}(\mathrm{bc}) \mathrm{d} & \mathrm{A} \rightarrow \mathrm{B} \vdash(\mathrm{C} \rightarrow \mathrm{A}) \rightarrow(\mathrm{C} \rightarrow \mathrm{B}) \\
\operatorname{Rabc} \Rightarrow \operatorname{Rbac} & \mathrm{A} \vdash(\mathrm{A} \rightarrow \mathrm{B}) \rightarrow \mathrm{B} \\
\mathrm{a} \sqsubseteq \mathrm{a}^{* *} & \mathrm{~A} \vdash \sim \sim \mathrm{A} \\
\mathrm{a}^{* *} \sqsubseteq \mathrm{a} & \sim \sim \mathrm{A} \vdash \mathrm{A} \\
\operatorname{Rabc} \Rightarrow \mathrm{Rac}^{*} b^{*} & \mathrm{~A} \rightarrow \mathrm{B} \vdash \sim \mathrm{B} \rightarrow \sim \mathrm{A} \\
\hline \text { Raaa } & \mathrm{A} \wedge(\mathrm{A} \rightarrow \mathrm{B}) \vdash \mathrm{B} \\
\mathrm{g} \sqsubseteq \mathrm{a} & \mathrm{A} \vdash \mathrm{B} \rightarrow \mathrm{A}
\end{array}
$$

In the first condition we use ' $R(a b) c d$ ' as a shorthand for ' $(\exists x)(R x c d \wedge R a b x)$ ' and ' $\operatorname{Ra}(b c) d$ ' for ' $\exists x)(\operatorname{Raxd} \wedge \operatorname{Rbcx})$.'

The result of adding all the conditions above the line is the substructural logic $C$, which is $R$ without the contraction axiom $A \wedge(A \rightarrow B) \vdash B$. Adding this gives us $R$, and adding instead the 'irrelevance' condition $A \vdash B \rightarrow A$ we get the logic $C K$ which is one of the strongest logics without the contraction axiom. For more details on these substructural logics, see [I5].

If we wish to model logics without negation, then we simply rid our frame of $*$ and its conditions. Models for positive $\mathrm{C}, \mathrm{R}$ and $\mathrm{CK}$ are given by the corresponding classes of frames.

We can extend these models to interpret quantifiers, by means of a constant domain interpretation. In the case of models of arithmetic with the $\omega$ rule our job is even easier. We may use a substitutional interpretation, as we have numerals as names for each object in the domain. We have the simple rules:

- $a \Vdash \forall x A(x)$ if and only if $a \Vdash A(n)$ for each $n$.

- $a \Vdash \exists x A(x)$ if and only if $a \Vdash A(n)$ for some $n$.

Predicates then are modelled by their extension at each point in the model, and function symbols are modelled by functions on the domain, as before. The models which result are models of the quantified logics C, R and CK. All of the 
theses of these logics are true at the base point $g$ of the models appropriate for those logics. We will use these frames to construct models for the arithmetics $\mathrm{C}^{\sharp \sharp}, \mathrm{R}^{\sharp \sharp}$ and $\mathrm{CK}^{\sharp \sharp}$.

\section{THE BEHAVIOUR OF IDENTITY}

A close inspection of the axioms reveals axioms of two different kinds. First, there are those which are identities. Second, there are axioms which are at heart, implications. The only rule left is the omega rule, which is dealt with by our taking the domain of each of our frames to be the natural numbers. Let us consider the identity axioms. These are as follows (free variables are implicitly universally quantified)

$$
\begin{gathered}
0=0 \quad x+0=x \quad x+y^{\prime}=(x+y)^{\prime} \\
x 0=0 \quad x y^{\prime}=x y+x \quad 0 \neq x^{\prime}
\end{gathered}
$$

These axioms are settled in our models by the behaviour of the identity relation at the base point $\mathrm{g}$, and by the interpretation of the functions. Since it is our aim to make these models look as much like standard arithmetic as possible except for the interpretation of the conditional, we take the interpretation the constant 0 to be the number zero, and the interpretation of the functions symbols for successor, addition and multiplication to be the functions addition, successor and multiplication on the domain of natural numbers. Finally, the extension of the identity relation at the base point is the standard identity relation on the natural numbers. This ensures that each of these axioms are dealt with. Except one. We have not ensured that $0 \neq x^{\prime}$, as this depends not on what happens at the base point, but at the point $g^{*}$. This will have to wait for a few sections for when we discuss negation.

The interest in these models of substructural arithmetic is not what happens at the base point $g$, but what happens elsewhere. This is what you would expect. The aim of substructural arithmetic is not to collect together a deviant set of arithmetic facts but rather to explain different connections between the classical arithmetic facts. The conditional provides the novelty, and this is reflected by the fact that the non-standard evaluation occurs on different points of the frame. These points can be considered to be different information states, which give us the tools to extract the connections between different arithmetic facts-what would happen in a hypothetical (but of course still impossible) situation in which $0=2$ helps us see what follows from identifying $\mathrm{o}$ and 2.

Let us see what models must satisfy in order to be models of substructural Peano arithmetic. The implication axioms are simple.

$$
\begin{gathered}
x=y \rightarrow y=x \quad x=y \rightarrow(y=z \rightarrow x=z) \\
x=y \rightarrow x^{\prime}=y^{\prime} \quad x^{\prime}=y^{\prime} \rightarrow x=y
\end{gathered}
$$


Let's take these one at a time to see how we can make them hold in our models. The first states, in the language of frames, that the extension of the identity predicate at each point is symmetric. For if $a \Vdash n=m$ then $a \Vdash m=n$ too.

The second axiom connects the extension of identity at different points related by R. In particular, we have the following condition:

$$
\text { Whenever Rabc, if } \mathrm{a} \Vdash \mathrm{n}=\mathrm{m} \text { and } \mathrm{b} \Vdash \mathrm{m}=\mathrm{l} \text { then } \mathrm{c} \Vdash \mathrm{n}=\mathrm{l} \text {. }
$$

We will call this the R-transitivity of identity. We do not so much require that identity be transitive at individual points, but we do require R-transitivity.

In the presence of the contraction condition for the logic R, R-transitivity entails standard transitivity, since Raaa for each $a$. If $a \Vdash n=m$ and $a \Vdash m=l$ then we must have a $\Vdash n=l$ too. So, in $R$, identity is transitive at each point in the frame. In the presence of the irrelevance condition for CK, we do not necessarily have transitivity, but since $\mathrm{g} \sqsubseteq \mathrm{a}$ for each $\mathrm{a}$, identity must be reflexive at each point, as identity is reflexive at $\mathrm{g}$.

The last two axioms connect identity at each point with the behaviour of the successor function. If $a \Vdash n=m$ then we must have $a \Vdash n^{\prime}=m^{\prime}$, and conversely, if $a \Vdash n^{\prime}=m^{\prime}$ then $a \Vdash n=m$ too. This means that the extension of identity at the point a must act like a congruence relation under successor. So at each point, the extension of identity must be symmetric, and it must be what we call a weak congruence with respect to successor. It is to the study of these symmetric weak congruences that we will now turn.

\section{SYMMETRIC WEAK CONGRUENCES AND FRAMES}

A two place relation $\rho$ on the set of natural numbers is a symmetric weak congruence if and only if it is symmetric, and if $n \rho m$ if and only if $n^{\prime} \rho m^{\prime}$ for each number $n$ and $m$. Symmetric weak congruences are rather simple structures, as can be seen by the following equivalence.

FACT I The trace Trace $\rho$ of a symmetric weak congruence $\rho$ is the set of all numbers $n$ such that $0 \rho n$. The congruence $\rho$ is determined uniquely by its trace Trace $_{\rho}$. And conversely, any set $X$ of numbers is the trace of some symmetric weak congruence $\rho_{X}$.

The fact is quite simple to prove. If $n \rho m$ then $|n-m|$, the absolute value of the difference $n-m$ is in the trace Trace $e_{\rho}$. Conversely if $n \in$ Trace $_{\rho}$ then $m \rho(n+m)$ and $(n+m) \rho m$ for each number $m$.

FACT 2 The weak congruence $\rho$ is reflexive if and only if $0 \in$ Trace $_{\rho}$. It is transitive if and only if whenever $m, n \in$ Trace $_{\rho}$ then $m+n,|m-n| \in$ Trace $_{\rho}$.

The first part of this fact is immediate. The second is almost as immediate. We have $m \rho n$ and $n \rho l$ if and only if $|m-n|,|n-l| \in$ Trace $_{\rho}$. Similarly, $m \rho l$ if and only if $|m-l| \in \operatorname{Trace}_{\rho}$. However $|m-l|$ is either equal to $|m-n|+|n-l|$ if $n$ is between $n$ and $l$, and it is ||$m-n|+| n-l||$ otherwise. 
So, points in models for substructural arithmetics have corresponding symmetric weak congruences, the extension of identity at these points.

We can take the correspondence in reverse too. We can construct a frame out of symmetric weak congruences. The identity relation on natural numbers, with trace $\{0\}$, can be the base point in this frame. The congruences are related by the following ternary relation $R$

$R \rho \lambda \chi$ if and only if whenever $n \rho m$ and $m \lambda l$, then $n \chi l$.

A corresponding condition on traces is simple: if $n \in X$ and $m \in Y$ then $n+m$ and $|n-m| \in Z$. This ternary relation is modelled explicitly after the connection between $R$ and the extension of identity in any model for substructural logics.

Congruences are also ordered by an inclusion relation $\sqsubseteq$

$$
\rho \sqsubseteq \lambda \text { if and only if whenever } n \rho m \text { then } n \lambda m \text {. }
$$

This corresponds to the subset relation on traces.

Then congruence relations, together with $R$ and $\sqsubseteq$ provide all the machinery for frames of the logic $\mathrm{C}$ without negation.

FACT 3 The class of symmetric weak congruence relations, ordered by $\sqsubseteq$ and related by $R$, is a positive $C$ frame. The class of transitive symmetric weak congruence relations is a positive $\mathrm{R}$ frame, and the class of reflexive symmetric weak congruence relations is a positive $\mathrm{CK}$ frame.

There are many requirements to check. The tonicity condition falls out of the definitions. The symmetry condition $\mathrm{Rabc} \Rightarrow \mathrm{Rbac}$ follows from the symmetry of the congruence relations. The associativity condition is the most tedious to check, but it is a restatement of a known fact of relation algebra: the associativity of relational composition. Note that R $\rho \gamma \chi$ if and only if $(\rho \gamma) \subseteq \chi$, where $(\rho \gamma)$ is the relation defined by setting

$$
n(\rho \gamma) l \text { iff there is some } m \text { where } n \rho m \text { and } m \gamma l
$$

Then, the associativity condition $R(a b) c d \Rightarrow R a(b c) d$ is equivalent to checking the associativity fact

$$
(\rho(\gamma \chi)) \sqsubseteq((\rho \gamma) \chi)
$$

for relational composition, and this is an immediate consequence of the definition of composition.

The identity point $g$ in a frame is the identity relation: $(g \rho)=\rho$ for any relation $\rho$.For $\mathrm{R}$, we have $\mathrm{R} \rho \rho \rho$, as the congruence relations are transitive: $(\rho \rho) \sqsubseteq \rho$. For CK, we have $\mathrm{g} \sqsubseteq \rho$ as congruence relations are reflexive.

So, frames constructed out of weak congruence relations give us models for our logics (without negation). It is only a short step to make these models of the 
entire language of arithmetic (except for the axiom involving negation). We take the domain to be the set of natural numbers, and we evaluate the identity predicate in the obvious way:

- $\rho \Vdash n=m$ if and only if $n \rho m$.

We interpret the function symbols in their standard way on the domain of natural numbers. This gives us a model.

FACT 4 This evaluation gives us a model of all of the positive axioms of Peano arithmetic.

The demonstration of this fact involves running our argument thus far in reverse. The symmetry of the relations gives us the symmetry axiom $x=y \rightarrow$ $y=x$. The definition of the relation $R$ gives us $x=y \rightarrow(y=z \rightarrow x=z)$. The fact that these are congruence relations ensures that $x=y \leftrightarrow x^{\prime}=y^{\prime}$, and the behaviour of the functions ensures that each identity axiom (for addition and multiplication) are satisfied at the base point $\mathrm{g}$, as identity is interpreted there in the normal fashion.

These models, for the positive parts of $\mathrm{C}^{\sharp \sharp}, \mathrm{R}^{\sharp \sharp}$ and $\mathrm{CK}^{\sharp \sharp}$, are genuinely substructural. In each model $\mathrm{g} \| 0=2 \rightarrow 0=1$. Take the congruence relation $\rho$ with trace $\{0,2,4, \ldots\}$. We have $\rho \Vdash 0=2$ but $\rho \Vdash 0=1$. This relation is symmetric, reflexive and transitive, so it appears in models for each of $C^{\sharp \sharp}, R^{\sharp \sharp}$ and $\mathrm{CK}^{\sharp \sharp}$. In fact, we have $\mathrm{g} \forall 0=\mathrm{m} \rightarrow 0=\mathrm{n}$ whenever $\mathrm{m}$ does not divide $\mathrm{n}$, as the trace $\{0, m, 2 m, 3 m, \ldots\}$ gives us a congruence relation in which $0=m$ holds but $0=n$ does not.

These models are also, to some degree, natural. The base point g models the arithmetic facts, and the other points are hypothetical arithmetic situations, in which the arithmetic truths are varied. The range of possible variations are constrained by the arithmetic laws such as the symmetry of identity. The degree of permissible variations establishes the connections between arithmetic claims. We have $0=2 \rightarrow 0=4$ in $\mathrm{R}$ arithmetic, since identifying 0 and 2 brings with it the identification of 0 and 4 by the transitivity of identity. We have $0=2 \leftrightarrow 2=4$, and $0=2 \rightarrow(2=4 \rightarrow 0=4)$, so $0=2 \rightarrow(0=2 \rightarrow 0=4)$ and by contraction, $0=2 \rightarrow 0=4$. This is reflected in our models by the behaviour of the appropriate congruences. Once $0 \rho 2$ we must have $0 \rho 4$ by transitivity. In logics without contraction, in which the number of times antecedents are used is important, we do not have $0=2 \rightarrow 0=4$. The most we have is $0=2 \rightarrow(0=2 \rightarrow 0=4)$. Our model provides a counterexample to $0=2 \rightarrow 0=4$, as the congruence with trace $\{2\}$ (or $\{0,2\}$, for CK) does not contain 4 , and so, we have a point at which $0=2$ holds but $0=4$ fails.

So, these models exhibit quite a degree of genuinely substructural behaviour. Unfortunately, as they stand, these models do not support negation. Remedying this is the focus of the last section of this paper. 


\section{ADDING NEGATION}

The structure of weak congruence relations is quite rich, but is not rich enough to model negation. Let's focus on the logic $\mathrm{R}$ to see why. Consider the traces of symmetric and transitive weak congruences. The identity trace is $\{0\}$. There is also the empty trace $\emptyset$. Every other transitive trace must contain 0 , since if $n \in$ Trace $_{\rho}$, we have $0 \rho n$ and $n \rho 0$, so by transitivity, $0 \rho 0$. So, $0 \in$ Trace $_{\rho}$. If $\{0\}$ is the identity trace, and if we wish the theory at $\{0\}$ (the set of claims which are taken to be true at $\{0\}$ ) to be complete, then almost every point in our model will be complete, as every for every point $a$, other than the empty set, we have $\{0\} \sqsubseteq a$. This is a problem when it comes to modelling negation.

To see why, we need to understand a little more about how the $*$ operator works. The operator $*$ takes points in frames to their informational "dual". The negation, $\sim A$ is true at a just when $A$ does not hold at $a^{*}$. When $a \neq a^{*}$, the way is open for $A \wedge \sim A$ to be true at $A$, or for $A \vee \sim A$ to fail at $a$. This makes the semantics of substructural logics quite flexible. 7 The operator $*$ interacts with inclusion in the obvious, order inverting way. If $a \sqsubseteq b$, then we must have $\mathrm{b}^{*} \sqsubseteq \mathrm{a}^{*}$. A point $\mathrm{a}$ is consistent and complete just when $\mathrm{a}=\mathrm{a}^{*}$ (then, negation at a behaves classically). It is said to be consistent if $a \sqsubseteq a^{*}$, for then $a \| A \wedge \sim A$. It is said to be complete if $a^{*} \sqsubseteq a$, for then, $a \Vdash A \vee \sim A$ for each $A$. If $*$ is of period two (that is, if $a^{* *}=a$ for each $a$ ) then $a$ is complete, $a^{*}$ is consistent, and vice versa.

The following result is a straightforward consequence of our definitions:

FACT 5 If $\mathrm{a} \sqsubseteq \mathrm{b}$ and $\mathrm{a}$ is consistent and complete, then $\mathrm{b}$ is consistent only when $\mathrm{a}=\mathrm{b}$ (for $\mathrm{b}^{*} \sqsubseteq \mathrm{a}^{*}=\mathrm{a} \sqsubseteq \mathrm{b}$, so $\mathrm{b} \sqsubseteq \mathrm{b}^{*}$ ensures that $\mathrm{b}=\mathrm{a}$ ). Similarly, if $\mathrm{b} \sqsubseteq \mathrm{a}$ and $\mathrm{a}$ is consistent and complete, then $\mathrm{b}$ is complete only when $\mathrm{a}=\mathrm{b}$.

In our model of symmetric transitive weak congruences, we would like the base point $g$ to be consistent and complete. It follows that $\emptyset$ is consistent, but not complete, and every other point is complete, but not consistent. As there are many symmetric transitive weak congruences extending the identity, it follows that no $*$ operation of period two can be defined on this class. If it could, then $\{0,2,4, \ldots\}^{*}$ and $\{0,3,6, \ldots\}^{*}$ would both be $\emptyset$. Which do you choose for $\emptyset^{*}$ ?

There is no doubt that this class of congruences has too few incomplete points to be a frame for R. Let's explore one way to attempt to remedy this defecit. (It will turn out to fail, but the manner in which it fails will be instructive, and will point to another model construction.)

Consider theories: sets of formulas closed under logical consequence. The set of formulas true at a point in a reduced $\mathrm{R}$ model is a theory. If $\mathrm{T}$ is an $\mathrm{R}$-theory, then so is $\mathrm{T}^{*}=\{\mathrm{A}: \sim \mathrm{A} \notin \mathrm{T}\}$. We will call this theory the double of $\mathrm{T}$.

\footnotetext{
${ }^{7}$ But of course, it makes the interpretation of the operator $*$ rather difficult. I will not engage in this discussion here. For more on the interpretation of negation in relevant logics and the operator $*$, I refer the reader to papers by Meyer and Martin [8], Dunn [4] and Restall [14].
} 
Consider now the theory $T$ given by adding $0=2$ to the set of $\mathrm{R}^{\sharp \sharp}$ truths and closing under consequence. This theory contains $0=2,0=4$, and $1=3$ and so on, together with $0=0,0 \neq 1,0 \neq 2$ and other arithmetic truths. We have $0 \neq 0$ since transitivity gives $0=2 \rightarrow(0=0 \rightarrow 0=2)$, so by contraposition, $0=2 \rightarrow(0 \neq 2 \rightarrow 0 \neq 0)$ and modus ponens gives $0 \neq 0$ as well.

Consider the double $T^{*}$. It doesn't contain $0=0$ (as our original theory has $0 \neq 0)$ or $0=1(T$ contains $0 \neq 0)$ or $0=2(T$ contains $0 \neq 2)$. It does contain $0 \neq 1$ and $0 \neq 3$ etc. (as $T$ does not contain $0=1,0=3$ ). The result is as follows

$$
\begin{array}{r}
\{0=0,0 \neq 0,0 \neq 1,0=2,0 \neq 2,0 \neq 3, \ldots\} \subset \mathrm{T} \\
\{0 \neq 1,0 \neq 3,0 \neq 5, \ldots\} \subset \mathrm{T}^{*}
\end{array}
$$

Similarly, if we consider the theory $S$ given by adding $0=3$, it and its double look like this:

$$
\begin{array}{r}
\{0=0,0 \neq 0,0 \neq 1,0 \neq 2,0=3,0 \neq 3,0 \neq 4, \ldots\} \subset S \\
\{0 \neq 1,0 \neq 2,0 \neq 4,0 \neq 5, \ldots\} \subset S^{*}
\end{array}
$$

The theories $\mathrm{S}^{*}$ and $\mathrm{T}^{*}$ are genuine $\mathrm{R}^{\sharp}$ theories (they are closed under all of the $\mathrm{R}^{\sharp \sharp}$ implications). They both contain $n o$ identities. However, they are different. They are different not in which identities they assert, but in which identities they rule out. Our models so far do not provide any way of reflecting this situation. These different theories are mirrored only in the empty point $\emptyset$. To remedy this, we would hope to extend the $R^{\sharp \sharp}$ model of symmetric transitive weak congruences by replacing $\emptyset$ by a collection of new points: doubles of the existing points in our frame. However, to do this, we would need to define the inclusion relation and $R$ on both the original points and their doubles, in such a way as to continue to validate the conditions for an $\mathrm{R}$-frame.

Some of these conditions are straightforward to meet, and the others are not. Our target frame is made up of the collection of congruence relations on the class of natural numbers (non-empty symmetric transitive weak congruences are equivalence relations, and hence, congruences), together with a point $a^{\prime}$ for each congruence $a-$ except for $g$, which needs no double, since the aim of the exercise is to set $\mathrm{g}^{*}=\mathrm{g}$ so that what is true at $\mathrm{g}$ is consistent and complete. So, we define the operator $*$ to take congruences to their doubles and back.

$$
\mathrm{g}^{*}=\mathrm{g} \quad \mathrm{a}^{*}=\mathrm{a}^{\prime} \quad \mathrm{a}^{\prime *}=\mathrm{a}
$$

The inclusion relation places each of the doubles under each of the congruences. The doubles are ordered inversely.

$$
\mathrm{a}^{\prime} \sqsubseteq \mathrm{b} \text { always } \quad \mathrm{a} \sqsubseteq \mathrm{b}^{\prime} \text { never } \quad \mathrm{a}^{\prime} \sqsubseteq \mathrm{b}^{\prime} \text { iff } \mathrm{b} \sqsubseteq \mathrm{a}
$$

This ensures that $\mathrm{a} \sqsubseteq \mathrm{b}$ iff $\mathrm{b}^{*} \sqsubseteq \mathrm{a}^{*}$. Now, for ease of presentation, we shall suppose that for every relation $a$-including $g$-we have its double $a^{\prime}$. In the special case of $g$, we simply set $g^{\prime}=g$. The sole difference between ' and ${ }^{*}$ is 
that ${ }^{*}$ is a function on the entire set of relations and doubles, whereas ' picks out the set of doubles (the extra objects together with g), and the original congruence relations (except for $\mathrm{g}$ ) are not identical to $a^{\prime}$ for any $a$.

Now, to make this structure an $\mathrm{R}$ frame, we need to define the ternary relation $R$, and this is where the difficulties emerge. Some constraints are, again, relatively straightforward: the commutativity condition (if Rabc then $\mathrm{Rbac}$ ) and contraposition (if Rabc then $\operatorname{Rac}^{*} b^{*}$ ) impose some constraints on any definition of $R$.

$$
\mathrm{Rab}^{\prime} \mathrm{c}^{\prime} \Longleftrightarrow \mathrm{Rb}^{\prime} \mathrm{ac}^{\prime} \Longleftrightarrow \mathrm{Racb}
$$

Similarly, the fact that each shadow $\mathrm{a}^{\prime}$ is below $\mathrm{g}$, means that Rabc' holds only when Rabg (by tonicity). Now, for reflexive, transitive weak congruences a and $\mathrm{b}$, Rabg only when $\mathrm{a}=\mathrm{b}=\mathrm{g}$. But in any reduced R-frame, if Rggx we must have $x \sqsubseteq g$. So, we must have.

$$
\operatorname{Rabc}^{\prime} \Longleftrightarrow \mathrm{a}=\mathrm{b}=\mathrm{c}^{\prime}=\mathrm{g}
$$

The next choice which is settled is $R a^{\prime} b^{\prime} c$. In this case, if the result is to be a reduced $\mathrm{R}$ frame, we need $\mathrm{Ra}^{\prime} \mathrm{b}^{\prime} \mathrm{c}$ to hold whenever $\mathrm{Rgb}^{\prime} \mathrm{c}$ (by tonicity, since $\mathrm{a}^{\prime} \sqsubseteq \mathrm{g}$ ), and then, $\mathrm{Rgb}^{\prime} \mathrm{c}$ holds if and only if $\mathrm{b}^{\prime} \sqsubseteq \mathrm{c}$. But this holds always, since the doubles are under the congruences. So, we have

$$
\mathrm{Ra}^{\prime} \mathrm{b}^{\prime} \mathrm{c} \text { always. }
$$

These are the simple cases. The remaining cases are for $\mathrm{Rab}^{\prime} \mathrm{c}, \mathrm{Ra}^{\prime} \mathrm{bc}$ and $R a^{\prime} b^{\prime} c^{\prime}$. These should be interdefinable, since $R a b^{\prime} c$ iff $R b^{\prime} a c$ (by commutativity) iff $\mathrm{Rb}^{\prime} \mathrm{c}^{\prime} \mathrm{a}^{\prime}$ (by contraposition). The most striaghtforward constraint on the definition of $\mathrm{Ra}^{\prime} \mathrm{bc}$ (and hence on the other clauses) is that

$$
\mathrm{Ra}^{\prime} \mathrm{bc} \text { if either } \mathrm{b} \sqsubseteq \mathrm{c} \text { or } \mathrm{b} \sqsubseteq \mathrm{a}
$$

since if $\mathrm{b} \sqsubseteq c$ then $R g b c$ and hence $R a^{\prime} b c$ by tonicity. Similarly, if $b \sqsubseteq a$ then $R c^{\prime} b a$, and hence $R b c^{\prime} a$, and $R b a^{\prime} c$, giving $R a^{\prime} b c$. However, this provides a necessary condition for $\mathrm{Ra}^{\prime} \mathrm{bc}$ (and the other clauses), but not a sufficient one.

Unfortunately, no way of deining R satisfying these conditions also satisfies the associativity condition, that $R(a b) c d \Rightarrow R a(b c) d$. The crucial case is verifying that if $R\left(x y^{\prime}\right) z u$ then $R x\left(y^{\prime} z\right) u$. To see why this is the case, we need to examine the structure of $R$ a little more.

FACT 6 Every reflexive symmetric transitive weak congruence relation is determined uniquely by the smallest nonzero number (if any) related to zero. The trace $\rho$ is equivalent to the equivalence of natural numbers modulo $|\rho|$ for some number $\rho$ (or $\infty$ when $\rho$ is identity).

This is a straightforward piece of elementary number theory, and a consequence of Fact 2. Consider Trace $\rho$, and set $|\rho|$ to be its smallest nonzero member (and $\infty$ if there is none). By transitivity, $\rho$ identifies all of the numbers 
modulo $|\rho|$. To see that it cannot identify any more, notice that we cannot have $m \rho n$ where the distance between $m$ and $n$ is less than $|\rho|$, since if $m \rho n$, then $0 \rho|m-n|$, but $|\rho|$ was chosen as the smallest number identified with zero. There can be no pairs of numbers in the series related any closer than $|\rho|$ apart.

Now we can expose some more of the underlying nature of the accessibility relation in the original positive frame.

FACT 7 Among the reflexive, symmetric weak congruence relations, R $\rho \gamma \chi$ if and only if both $|\rho|$ and $\gamma \mid$ divide $|\chi|$. Also, among inclusion relations, $\rho \sqsubseteq \gamma$ if and only if $|\gamma|$ divides $|\rho|$.

In the statement of this fact, we have used the obvious convention that $n$ divides $\infty$, which corresponds to the fact that the identity relation is included in every trace. In what follows, we will use the notation ' $\rho_{n}$ ' to denote the relation of equivalence modulo $n$. Identity is $\rho_{\infty}$.

Now we can expose the failure of associativity in any frame consisting of these congruence relations and their doubles. As I mentioned, the crucial case is the step from $R\left(x y^{\prime}\right) z u$ to $R x\left(y^{\prime} z\right) u$. If $R\left(x y^{\prime}\right) z u$ then it follows that the intermediate point is either an original relation, or a shadow: we have either $R x y^{\prime} w$ and $R w z u$ (it is a congruence) or $R x y^{\prime} w^{\prime}$ and $R w^{\prime} z u$ (it is a shadow). What is supposed to follow from this is $R x\left(y^{\prime} z\right) u$. Again, the intervening point is either a congruence, or a shadow. If it is a congruence, we have $R y^{\prime} z v$ and $R x v u$. If it is a shadow, we have $R y^{\prime} z v^{\prime}$ and $R x v^{\prime} u$. In other words, what we require is the following condition:

If either (a) $R x y^{\prime} w$ and $R w z u$ or (b) $R x y^{\prime} w^{\prime}$ and $R w^{\prime} z u$ then there is some congruence $v$ such that either (i) $\mathrm{Ry}^{\prime} z v$ and $\mathrm{Rxvu}$ or (ii) $R y^{\prime} z v^{\prime}$ and $R x v^{\prime} u$.

Now, we do not know exactly when Rxy'w or Rw'zu holds, but we do know that if $x \sqsubseteq y$ then $R x y^{\prime} w$, and that if $z \sqsubseteq u$ then $R w^{\prime} z u$. In particular, if the required condition holds, then at the very least, we have

If either (a) $x \sqsubseteq y$ and $R w z u$ or (b) $R x y^{\prime} w^{\prime}$ and $z \sqsubseteq u$ then there is a congruence $v$ such that either (i) $R x v u$ or (ii) $R y^{\prime} z v^{\prime}$.

We have also discarded a clause from each of (i) and (ii) as they will play no part in what follows. Now we will rewrite the conditions in such that the shadows disappear. The remaining conditions can be expressed in terms of the congrences alone

If either (a) $x \sqsubseteq y$ and Rwzu or (b) Rxwy and $z \sqsubseteq u$ then there is a congruence $v$ such that either (i) Rxvu or (ii) Rzvy.

Now, let me abuse notation just a little, and stop considering the congrences $x, y, z$ etc., and consider now their periods. Instead of the congruence $x$, we have its period, which we shall also call ' $x$ '. Rewriting the condition, we have 
If either (a) $y|x, u| z$ and $u \mid w$ or (b) $y|x, y| w$ and $u \mid z$ then there is a some $v$ such that either (i) $u \mid x$ and $u \mid v$ (ii) $y \mid z$ and $y \mid v$.

This condition fails when $x=y=2, u=z=3$ and $w=6$. In this case we have both (a) $2|2,3| 3$ and $3 \mid 6$ (b) $2|2,2| 6$ and $3 \mid 3$, but neither (i) since $u=3 \nmid 2=x$ nor (ii) since $y=2 \nmid 3=z$.

FACT 8 There is no embedding of the R-frame of symmetric transitive weak congruences into an $\mathrm{R}$-frame consisting of the congruence relations and a double for each relation, satisfying $\mathrm{g}^{*}=\mathrm{g}$.

So, this quest for a negation complete and consistent $\mathrm{R}$ arithmetic has failed. The failure can point our way, however, to a different kind of success. Our accessibility relation $R$, at least on the original points of the frame (before adding the shadows) collapses into the inclusion relation, with the equivalence

$$
\mathrm{Rabc} \Longleftrightarrow \mathrm{a} \sqsubseteq \mathrm{c} \text { and } \mathrm{b} \sqsubseteq \mathrm{c}
$$

This is the characteristic frame condition corresponding to the mingle axiom.

$$
A \rightarrow(A \rightarrow A)
$$

Our frame for $\mathrm{R}$ without negation was in fact for a stronger logic-it validated $\mathrm{R}^{+}$together with mingle. This motivates attempting to define negation in another way: through the simple three-valued frames for RM due to Dunn [2, 3].

\section{CONSISTENT, COMPLETE RM ARITHMETICS}

Dunn's frames for RM are simple strucrures. Each RM frame is a totally ordered set of points $\langle\mathrm{P}, \sqsubseteq\rangle,\left.\right|^{8}$ The complexity required to interpret a substructural logic is found in the definition of satisfaction. The evaluation relation bifurcates into two relations $\Vdash^{+}$and $\Vdash^{-}$. The two relations $\Vdash^{+}$and $\Vdash^{-}$are an evaluation on an RM frame when they satsify the following conditions for completeness and heredity, relating points and atomic sentences:

- Either $a \Vdash^{+}$p or $a \Vdash^{-} p$.

- If $\mathrm{a} \sqsubseteq \mathrm{b}$ then if $\mathrm{a} \Vdash^{+} \mathrm{p}, \mathrm{b} \Vdash^{+} \mathrm{p}$ too. Similarly, if $\mathrm{a} \Vdash^{-} p$ then $\mathrm{b} \Vdash^{-} p$ too.

The relations encode positive and negative information. Positive and negative information are exhaustive (by the lights of RM, at least). And information that we grasp is preserved as we shift from one point to another, up the ordering. Completeness and heredity jointly entail either that every point is exactly the

\footnotetext{
${ }^{8}$ Compare the simplicity of this definition of a frame with the relative complexity of the definition of a reduced ternary frame with negation.
} 
same as far as information goes, or that as information is added, it becomes inconsistent.

An evaluation can be used to interpret the other connectives in a straightforward manner?

- $a \Vdash^{+} \sim A$ if and only if $a \Vdash^{-} A$.

- $a \Vdash^{-} \sim A$ if and only if $a \Vdash^{+} A$.

- $a \Vdash^{+} \mathrm{A} \wedge \mathrm{B}$ if and only if $\mathrm{a} \Vdash^{+} \mathrm{A}$ and $\mathrm{a} \Vdash^{+} \mathrm{B}$.

- $a \Vdash^{-} A \wedge B$ if and only if $a \Vdash^{-} A$ or $a \Vdash^{-} B$.

- $a \Vdash^{+} A \vee B$ if and only if $a \Vdash^{+} A$ or $a \Vdash^{+} B$.

- $a \Vdash^{-} A \vee B$ if and only if $a \Vdash^{-} A$ and $a \Vdash^{-} B$.

- $a \Vdash^{+} A \rightarrow B$ if and only if for each $b \sqsupseteq a$, (a) if $b \Vdash^{+} A$ then $b \Vdash^{+} B$, and (b) if $b \Vdash^{-} B$ then $b \Vdash^{-} A$.

- $\mathrm{a} \Vdash^{-} \mathrm{A} \rightarrow \mathrm{B}$ if and only if either (c) $\mathrm{a} \Vdash^{+} \mathrm{A}$ and $\mathrm{a} \Vdash^{-} \mathrm{B}$ or (d) $a \|^{+} \mathrm{A} \rightarrow \mathrm{B}$.

There are three distinctive features of these clauses. First, the conjunction, disjunction and negation clauses are what one would expect once we pull positive and negative information apart $\mathrm{I}$ Second, the components (a) and (b) in the positive clause for the conditional are required for the conditional $A \rightarrow B$ to support modus ponens and contraposition (we wish $A \rightarrow B$ to give us $\sim B \rightarrow \sim A$ ). Third, the components (c) and (d) in the negative clause for the conditional are required in order to validate the $R M$ inference from $A \wedge \sim B$ to $\sim(A \rightarrow B)$, and in order to ensure that completeness holds for conditional formulas.

Dunn proves [2] that completeness and heredity hold for all sentences, and not just atomic ones, and that at each point all theorems of $\mathrm{R}$ and the mingle axiom, are true (that is, if $A$ is a theorem of $R+$ mingle then $a \Vdash^{+} A$ in each $\mathrm{RM}$ model). The fact that $\sqsubseteq$ is a total order is required in the proof to ensure heredity. Without a total order, heredity may break down.

These models for RM allow us to model negation without requiring incomplete points. Even though $A \rightarrow B \vee \sim B$ is invalid in $R M$, we do not need an incomplete point to invalidate it. In our model we need merely to have a point $\mathrm{b}$ such that $\mathrm{b} \Vdash^{-} \mathrm{B} \vee \sim \mathrm{B}$ (by setting $\mathrm{b} \Vdash^{-} \mathrm{B}$ and $\mathrm{b} \Vdash^{+} \mathrm{B}$-we are inconsistent about $B$ at the point $b$ ) without $b \Vdash^{-} A$. This is straightforward, provided we can be inconsistent about $B$ without making $A$ false.

We need only to interpret quatifiers, and then we can construct our models for arithmetic. For quantifiers, we do the usual thing, with a fixed domain of quantification, the standard numbers:

\footnotetext{
${ }^{9}$ These clauses are taken directly from Dunn's original paper [2], with only a change in notation.

${ }^{\text {Io }}$ They are shared with Priest's logic LP, Nelson's constructive negation, and so on.
} 
- $a \Vdash^{+} \forall x A(x)$ if and only if $a \Vdash^{+} A(n)$ for each $n$.

- $a \Vdash^{-} \forall x A(x)$ if and only if $a \Vdash^{-} A(n)$ for some $n$.

- $a \Vdash^{+} \exists x A(x)$ if and only if $a \Vdash^{+} A(n)$ for some $n$.

- $a \Vdash^{-} \exists x A(x)$ if and only if $a \Vdash^{-} A(n)$ for each $n$.

Now, we have all the raw materials to define a class of models for $\mathrm{RM}^{\sharp \sharp}$.

FACT 9 Any subset of numbers of $\{1,2, \ldots, \infty\}$ totally ordered by the relation of divisibility determines a model for $\mathrm{RM}^{\sharp \sharp}$, in which the points are the congruence relations $\rho_{n}$ for the chosen values of $n$, for which we set $\rho_{n} \sqsubseteq \rho_{m}$ if and only if $m$ divides $n$, and

- $\rho_{\mathrm{n}} \Vdash^{+} l=\mathrm{m}$ if and only if $l \equiv \mathrm{m}(\bmod \mathrm{n})$.

- $\rho_{\mathrm{n}} \Vdash^{-} l \neq m$ if and only if $l \neq m$, or $n \neq \infty$.

We require the total ordering of congruences in order for this to be an RM frame. Heredity on atomic relations is given by the definition of ordering: if $l \equiv \mathrm{m}(\bmod n)$ and $\rho_{n} \sqsubseteq \rho_{n}^{\prime}$, then $n^{\prime}$ divides $n$, so we have $l \equiv m\left(\bmod n^{\prime}\right)$ too. Negative information changes less. At the consistent point $\rho_{\infty}$, only actually distinct numbers are taken to be distinct. At all other points, even identical numbers are taken to be distinct. So heredity holds here. Completeness is immediate, since at the point $\rho_{\infty}, l=m$ is true if and only if it is not false. At all other points, more obtains, not less, so completeness is not violated anywhere. The result is a model for $\mathrm{RM}^{\sharp \sharp}$ by the usual reasoning.

Now, consider a model in which we include the point $\rho_{2}$. At $\rho_{2}$ we have $0=2$, but we do not have $0=3$. So, at any $\rho_{2}$ and earlier point in the model, we do not have $0=2 \rightarrow 0=3$. We have $\rho_{2} \Vdash^{+} \sim(0=2 \rightarrow 0=3)$, since $\rho_{2} \not \nvdash^{+} 0=2 \rightarrow 0=3$ because $\rho_{2} \Vdash^{+} 0=2$ and $\rho_{2} \|^{+} 0=3$.

Similarly, if our model contains $\rho_{3}$, we have $\sim(0=3 \rightarrow 0=2)$ holding at $\rho_{3}$ and at every earlier point in the model. However, no RM model contains both $\rho_{2}$ and $\rho_{3}$, since 2 doesn't divide 3 and 3 doesn't divide 2 . Nonetheless, we can go fairly far.

FACT IO The model corresponding to a subset of numbers $\{1,2, \ldots, \infty\}$ containing $\infty$ determines a consistent complete model of $\mathrm{RM}^{\sharp \sharp}$. Whenever $\rho_{n}$ is a point in the model and $0 \not \equiv m(\bmod n)$, then at $\rho_{\infty}$ in this model we have $\sim(0=\mathrm{n} \rightarrow 0=\mathrm{m})$.

The point $\rho_{\infty}$ is consistent and complete. It is consistent for atomic formulas by construction. The fact that is is consistent for all formulas is a straightforward induction on the complexity of formulas. Since in our model $\rho_{\mathrm{n}} \Vdash^{+} 0=\mathrm{n}$ but $\rho_{\mathrm{n}} \not \nvdash^{+} 0=\mathrm{m}$ whenever $0 \not \equiv \mathrm{m}(\bmod \mathrm{n})$, we have $\rho_{\infty} \forall^{+} 0=$ $\mathrm{n} \rightarrow 0=\mathrm{m}$, and hence $\rho_{\infty} \Vdash^{+} \sim(0=\mathrm{n} \rightarrow 0=\mathrm{m})$ as desired. 
So, we have at last found consistent and complete models of arithmetic in $\mathrm{R}$ - actually, models in RM-in which irrelevant implications between identities are denied. We can model $\sim(0=2 \rightarrow 0=3)$ (provided we include $\left.\rho_{2}\right)$. And $\sim(0=3 \rightarrow 0=2)$ (provided we include $\left.\rho_{3}\right)$. Unfortunately, we cannot model both.

\section{CONCLUSION}

These constructions open up more questions than they answer. Is there a consistent and complete model of $\mathrm{R}^{\sharp \sharp}$ in which all irrelevant conditionals between identities are denied? In each of the models we have examined, is there a simple axiomatisation of the claims true at $g\left(=\rho_{\infty}\right)$ ? What else can we say about models in the logics $\mathrm{C}$ and CK? The symmetry of weak congruences is tied up with the symmetry axiom $x=y \rightarrow y=x$. Why not reject symmetry? (In the absence of contraction we have rejected transitivity of identity at each point, after all.) Is there any way we can keep symmetry of identity at the base point without having symmetry everywhere else? Does rejecting symmetry give us models of weaker substructural logics? What about other mathematical theories? Do these models tell us anything useful about the behaviour of identity in substructural logics?

\section{REFERENCES}

[I] Alan ross anderson, NUEl D. Belnap, AND J. Michael DUNn. Entailment: The Logic of Relevance and Necessity, volume 2. Princeton University Press, Princeton, 1992.

[2] J. Michael DunN. "A Kripke-style semantics for R-mingle using a binary accessibility relation”. Studia Logica, 35:163-172, 1976.

[3] J. Michael dunn. "Quantification and RM". Studia Logica, 35:315-322, 1976.

[4] J. Michael dunn. "Star and Perp: Two Treatments of Negation". In James e. tomber lin, editor, Philosophical Perspectives, volume 7, pages 33I-357. Ridgeview Publishing Company, Atascadero, California, 1994.

[5] RовеRт K. MEYer. "Relevant arithmetic". Bulletin of the Section of Logic, $5: 133-137,1976$.

[6] robert K. meyer. "Arithmetic Formulated Relevantly". Unpublished manuscript. Some of the results appear in Entailment Volume 2 [I], 1979.

[7] robert K. Meyer. " $\supset \mathrm{E}$ is Admissible in 'True' Relevant Arithmetic”. Fournal of Philosophical Logic, 27:327-351, 1998.

[8] robert k. meyer and errol p. martin. "Logic on the Australian Plan". fournal of Philosophical Logic, 15:305-332, 1986.

[9] Robert K. meyer and Chris mortensen. "Inconsistent Models for Relevant Arithmetics". Fournal of Symbolic Logic, 49:917-929, 1984. 
[io] robert k. meyer and greg Restall. "Strenge' Arithmetic". Logique et Analyse, 42:205-220, 1999 (note: this paper was published in 2002).

[ir] Chris mortensen. Inconsistent Mathematics. Kluwer Academic Publishers, 1995.

[I2] GREg RESTALL. "Arithmetic and Truth in Łukasiewicz's Infinitely Valued Logic". Logique et Analyse, I39-I40:303-312, 1992.

[13] Greg restall. OnLogics Without Contraction. PhD thesis, The University of Queensland, January 1994 .

[I4] GREg RESTALL. "Negation in Relevant Logics: How I Stopped Worrying and Learned to Love the Routley Star". In DOV GabBAY AND HEINRICH WANSING, editors, What is Negation?, volume I3 of Applied Logic Series, pages 53-76. Kluwer Academic Publishers, 1999.

[15] GREg ReSta Ll. An Introduction to Substructural Logics. Routledge, 2000.

[i6] richard Routley and robert k. Meyer. "Semantics of Entailment - i I". The fournal of Philosophical Logic, I:53-73, 1972.

[I7] Richard ROUtley aNd ROBERT K. MEyer. "Semantics of Entailment". In hugues leblanc, editor, Truth, Syntax and Modality, Proceedings of the Temple University Conference on Alternative Semantics, pages 194-243. North Holland, I973. 
The Australasian fournal of Logic (ISSN I448-5052) disseminates articles that significantly advance the study of logic, in its mathematical, philosophical or computational guises. The scope of the journal includes all areas of logic, both pure and applied to topics in philosophy, mathematics, computation, linguistics and the other sciences.

Articles appearing in the journal have been carefully and critically refereed under the responsibility of members of the Editorial Board. Only papers judged to be both significant and excellent are accepted for publication.

The journal is freely available at the journal website at

http://www .philosophy . unimelb.edu.au/ajl/

All issues of the journal are archived electronically at the journal website.

SUBSCRIPTIONS Individuals may subscribe to the journal by sending an email, including a full name, an institutional affiliation and an email address to the managing editor at ajl-editors@unimelb.edu.au Subscribers will receive email abstracts of accepted papers to an address of their choice. For institutional subscription, please email the managing editor at ajl-editors@unimelb.edu.au.

Complete published papers may be downloaded at the journal's website at http://www.philosophy.unimelb.edu.au/ajl/ The journal currently publishes in pdf format.

Submission The journal accepts submissions of papers electronically. To submit an article for publication, send the ${ }^{\Delta} \mathrm{T}_{\mathrm{E}} \mathrm{X}$ source of a submission to a member of the editorial board. For a current list of the editorial board, consult the website.

The copyright of each article remains with the author or authors of that article. 